\title{
Check-list of Veronica subg. Pseudolysimachium (Plantaginaceae) of Siberia
}

\section{Конспект вероник подрода Pseudolysimachium (Veronica, Plantaginaceаe) Сибири}

\author{
P. A. Kosachev' ${ }^{1}$ D. Albach², A. L. Ebel ${ }^{3}$ \\ П. А. Косачёв ${ }^{1}$, Д. Альбах ${ }^{2}$, А. Л. Эбель ${ }^{3}$ \\ ${ }^{1}$ Altai State University, Lenina str., 61, Barnaul, 656049, Russia.E-mail: pakosachev@yandex.ru \\ ${ }^{1}$ Алтайский государственный университет, пр-т Ленина, 6,; Барнаул, 656049, Россия \\ ${ }^{2}$ Institute of Biology and Environmental Sciences, Carl von Ossietzky University of Oldenburg, \\ Carl von Ossietzky-Str. 9-11, Oldenburg, 26111, Germany \\ ${ }^{2}$ Институт биологии и наук о Земле, Университет им. Карла фон Осиикого, ул. Карла фон Осиџкого, \\ 9-11, Ольденбург, 26111, Германия.E-mail: dirk.albach@uni-oldenburg.de. \\ ${ }^{3}$ Tomsk State University, Lenina str.,36,Tomsk, 634050,Russia.E-mail: alex-08@mail2000.ru \\ ${ }^{3}$ Томский государственный университет, пр. Ленина, 36, Томск, 634050, Россия
}

Key words: systematic, hybrids, Asia, conspectus, Scrophulariaceae s. 1., key to species.

Ключевые слова: систематика, гибриды, Азия, конспект, Scrophulariaceae s. 1., ключ для определения видов.

Summary. Here, we present a critical check-list of Veronica subgenus Pseudolysimachium (W. D. J. Koch) Buchenau (Plantaginaceae Juss.) in Siberia, represented by 4 sections, 19 species and 4 subspecies. Two subsections of the subgenus (Pinnataе и Dauricae) are elevated to the taxonomic rank of a section. The Latin diagnosis for $V$. linariifolia var. baicalensis Boriss. is given, which has been previously described only in Russian.

Аннотация. В статье представлен критический конспект вероник подрода Pseudolysimachium (W. D. J. Koch) Buchenau (Plantaginaceae Juss.) Сибири, состоящий из 4 секций, 19 видов и 4 подвидов. Для двух подсекций (Pinnatae и Dauricae) предложен секционный таксономический ранг. Обнародовано название разновидности (V. linariifolia var. baicalensis Boriss.), ранее описанной на русском языке.

\section{Introduction}

Veronica subgenus Pseudolysimachium (W. D. J. Koch) Buchenau (Scrophulariaceae Juss. s. 1.; Plantaginaceae Juss., following APG II (2003) and III (2009)) is a distinct (with the exception of $V$. schmidtiana Regel) and easily recognizable group in the genus distributed across Eurasia (Albach et al., 2004).

In the last decade renewed interest has been given to the study of the subgenus. This interest has been raised by the polymorphism and frequent hybridization and polyploidy in the group. This has led to considerable systematic complexity in the group and many taxonomic questions. Based on phylogenetic studies based on nuclear and plastid DNA regions, the relationship of the subgenus within the genus has been clarified (Albach, Chase, 2001; Albach, Fischer, 2003; Albach et al., 2004, 2005). However, relationships within the subgenus remain obscure.

In recent years, the renewed interest has led to studies of comparative morphology and ecogeography of the species in the Altai Mountains, which revealed unanticipated taxonomic diversity (Kosachev, 2003, 2010; Kosachev, German, 2004). Before these studies subgenus Pseudolysimachium was studied in Siberia only floristically in some regions. For the whole former USSR only a few 
more specialized but still regional studies in the group have been published (Afanasjeva, Meschkova, 1961; Elenevsky, 1968, 1971, 1977, 1978; Klokov, 1976; Manukjan, 1984; Tzvelev, 1981) despite the fact that an important role for the evolution of the subgenus has been assigned to the development of the Eurasian steppe across the former USSR (Kamelin, 1998). For the Altai territory a systematic overview was given by Kosachev (2003), which we update here based on our more recent studies using field observations, morphology, flow cytometry and DNA-based phylogenetic analyses.

\section{Key to the Veronica subgen.} Pseudolysimachium of Siberia

1. Leaves pinnate or pinnatepartite .. 2

+ Leaves entire, margin serrate, dentate, crenate or shallowly lobed

2. All leaves sessile, pinnately dissected into linear or filiform lobes. Calyx glabrous

+ Leaves with short or long petioles, all or only the lower leaf blades pinnately divided into oblong or lanceolate obtuse lobes. Calyx pubescent

3. Plant $15-50 \mathrm{~cm}$ tall. Leaves $1-3 \mathrm{~cm}$ long. Inflorescences long (up to $25 \mathrm{~cm}$ ), sparse. Calyx cut into lanceolate, long pointed lobes. Corolla pale blue, white or pink

13.1. V. pinnata L. subsp. pinnata

+ Plants 5-10 cm tall. Leaves $0.5-1 \mathrm{~cm}$ long. Inflorescences short $(1-1.5 \mathrm{~cm})$. Calyx divided into semi-ovate lobes. Corolla bright blue

..13.2. V. pinnata subsp. nana (Kryl.) Polozhij

4. All above-ground parts of the plant glandular pubescent 16. $\boldsymbol{V} . \times$ sessiliflora Bunge

+ Plants, except for mixed simple and glandular ovary and capsule, pubescent with simple hairs only

5. Plants to $45 \mathrm{~cm}$ tall, green. Inflorescences solitary, rarely branched. Calyx pubescent with short glandular hairs ...... 15. $\boldsymbol{V}$. $\times$ altaica Kosachev

+ Plants to $50(55) \mathrm{cm}$ tall. Inflorescence paniculate. Calyx pubescent with simple hairs

12. V. reverdattoi Krasnob.

6. All parts of the plant white-tomentose with simple, abundant curly hairs

+ Green or gray-green plants, not tomentose, with simple or glandular hairs ... 9

7. Leaf margins finely serrate-dentate. Vegetative shoots at the base of the generative shoot absent ....

8. V. sergievskiana Polozhij

+ Leaf margins shallowly crenate, upper leaves entire. Short vegetative shoots present at the base of the generative shoot
8. Calyx lobes lanceolate-linear, $3 \mathrm{~mm}$, with a few glandular hairs on the margin. Corolla with rounded-ovate, obtuse lobes. Stamens not exposed or nearly exposed from the corolla

11. $\boldsymbol{V} \times$ czemalensis Kosachev et Albach

+ Calyx lobes ovate-oblong or lanceolate, 3-4 $\mathrm{mm}$ long. Corolla with oblong-ovate to broadly ovate lobes. Stamens markedly exserted from corolla

7. V. incana L.

9. All parts of the plant pubescent with multicellular glandular hairs, making the plant in living state sticky 10. V. porphyriana Pavl.

+ Plants pubescent with simple and (or) short glandular hairs, if multicellular glandular hairs present, then middle and upper leaves deeply serrated ( $V$. sajanensis) ...................................... 10

10. Leaves deeply unequally or sharply, often twice serrate ..................................................... 11

+ Leaves crenate, finely serrate, shallowly serrate-dentate or entire 14

11. Leaves deeply serrate-dentate. Inflorescence axis, calyx, bracts and pedicels shaggy from dense pubescence of long simple and glandular hairs. Calyx almost to the base divided into very narrow, linear and acute lobes. Plants of subalpine and alpine meadows, less common in tundra

3. V. sajanensis Printz

+ Other combination of characters 12

12. Leaves covered with simple or a mixture of simple and glandular hairs, ovate or oblong-ovate, margin deeply unequally serrate

14. V. daurica $\mathrm{L}$.

+ Leaves glabrous or with a few sparse hairs, broadly lanceolate or lanceolate; margin serratedentate, incised serrate-dentate, often doubledentate

13. Plants to $60 \mathrm{~cm}$ tall, strongly branched above. Stems procumbent to ascending. Leaves deeply incised-dentate. Bracts long, exceeding the pedicel, at the top of the inflorescence almost reaching the top of the calyx teeth ..2. V. taigischensis Stepanov

+ Plants 50 to $120 \mathrm{~cm}$ tall, weakly branched above. Stems erect. Leaves serrate, often twicedentate. Bracts equal to pedicel or slightly longer .. .. 1. V. longifolia $\mathrm{L}$.

14. Leaves whorled, 3-4 in a whorl. Bracts shorter than pedicels 5. V. spuria L.

+ Leaves opposite, sometimes alternate in the upper part of the stem. Bracts reach middle of calyx or beyond 15

15. Leaves serrate or unequally serrate. Corolla lobes ovate, ovate-lanceolate, oblong-rounded, obtuse or slightly pointed 18 
+ Leaves crenate or slightly dentate. Corolla lobes lanceolate, long-acuminate .. 16

16. ........... Plants gray-green, hairy in all parts of glandular hairs with a slight admixture of simple hairs ....................... 9.1. V. spicata subsp. spicata

+ Plants dark green, sometimes glossy. Stems in lower half glabrous, in the upper half covered with short glandular hairs, or the stems completely glabrous 17

17. Plants glabrous; bracts up to $1 \mathrm{~cm}$ long .... 9.2. V. spicata subsp. kamelinii Kosachev

+ Plants glabrous below the middle, densely pubescent with glandular hairs above; bracts up to $0.5 \mathrm{~cm}$ long 9.3. V. spicata subsp. paczoskiana (Klok.) Kosachev

18. All parts of the plant densely covered with long white cobwebby hairs .. 19

+ Plants covered with short simple and glandular hairs. Sometimes leaves glabrous ( $V$. linariifolia) ..

19. Sepals almost triangular, $1.5-2 \mathrm{~mm}$ long. Capsule obovate, about $3 \mathrm{~mm}$ long and wide, with a wide triangular notch about $1.5 \mathrm{~mm}$ deep, glabrous 18. $\boldsymbol{V} \times$ grisea Kosachev et A. L. Ebel

+ Calyx lobes linear, $4 \mathrm{~mm}$ long. Capsule $2.5 \times$ $2 \mathrm{~mm}$, rounded-ovate, with no recesses, pubescent with long white hairs on the entire surface

4. V. krasnoborovii Kosachev et Schaulo

20. Leaves opposite, lower ovate to ovateoblong, with rounded or wedge-shaped base; middle and upper leaves oblong-lanceolate, with wedgeshaped base. Capsule at the top pubescent with simple, jointed hairs. Streamsides of upper belt of Altai 17. $\boldsymbol{V} \times$ × schmakovii Kosachev

+ Lower leaves opposite, upper ones can be alternate; oblong-lanceolate or narrowly lanceolate, cuneate at the base. Capsule pubescent at the top with glandular hairs or glabrous. Plants of steppe belt (bushes, slopes) 21

21. Stems hairy with 2-3-celled simple downwards curved hairs, in the inflorescence with short glandular hairs. Leaves on the margin with 2-3-celled glands. Capsule in the upper third covered with long simple and glandular hairs

19. $\boldsymbol{V}$. $\times$ kolyvanensis Kosachev et Shmakov

+ Stems covered with short simple hairs curved upwards. Leaves glabrous, rarely pubescent with scattered hairs. Capsule glabrous

6. V. linariifolia Pall. ex Link

In the process of assembling this critical conspectus we investigated material from the following herbariums ALTB, IRK, LE, MHA, MW,
NS, NSK, TK, and the herbarium of Altai botanical garden (Ridder, Kazakhstan).

In the conspectus we provide the main bibliographical information (regional floras and determination keys) that summarize the floristic knowledge of the investigated territory. We state all synonyms relevant for the territory of Siberia, as well as information on types, taxonomy, ecology, distribution area within Siberia and chromosome number/ploidy level. Distribution in the region follows the floristic districts of the "Flora of Siberia" (Malyshev, 1988).

\section{Conspectus of Veronica subgenus Pseudolysimachium Buchenau in the territory of Siberia}

Subgen. Pseudolysimachium (W. D. J. Koch) Buchenau, 1894, Fl. Nordwestd. Tiefebene: 447; Ortega, Albach, Fischer, 2008, Taxon, 57, 1: 3. $-V$. sect. Pseudolysimachium W. D. J. Koch, 1837, Syn. Fl. Germ.: 527 ("Pseudo-Lysimachium", see ICBN Art. 60.9); Borisova, 1955, Fl. USSR, 22: 367; Elenevsky, 1978, Sist. Geogr. Veronik SSSR Prilezh. Stran: 97; Polozhij, 1996, Fl. Sib. 12: 28; Assejeva, 2002, Novit. Syst. Pl. Vasc., 34: 160. - Gen. Pseudolysimachion Opiz, 1852, Seznam: 80; Hong et Fischer, 1998, Fl. China, 18: 66.

Perennial plants with terminal inflorescences (sometimes also lateral ones). Leaves opposite or verticillate. Calyx quadripartite. Corolla tube length longer than width, hairy inside, with four vascular bundles. Capsules mostly shorter than $4 \mathrm{~mm}$, flattened, almost elliptic or circular, apex obtuse or shortly notched. Style longer than capsule. Seeds numerous, small, flattened, reticulate. Base chromosome number $x=17$. Palearctic: Europe, Northern Asia, North America, in Japan south to tropical regions.

Lectotype (Holub, Pouzar, 1967): V. spicata L. (Pseudolysimachium spicatum (L.) Opiz).

1. Veronica sect. Longifoliae (Holub) Kosachev et Albach, comb. nova. - Pseudolysimachion sect. Longifoliae (Holub) Trávniček, 1998, Preslia, 70, 3: 195. - Pseudolysimachion ser. Longifoliae Holub, 1967, in Holub et Pouzar, Folia Geobot. Phytotax. 2, 4: 423. - Veronica subsect. Longifoliae (Holub) A. Jelen. 1977, Bull. Soc. Nat. Moscou, 82, 1: 151; Assejeva, 2002, Novit. Syst. Pl. Vasc., 34: 160, p. p. $V$. sect. Pseudolysimachia ser. Longifoliae Boriss. 1955, Fl. USSR, 22: 372 (descr. ross.). - Pseudolysimachion ser. Spuriae Holub, 1967, in Holub et Pou- 
zar, Folia Geobot. Phytotax. 2, 4: 423. - Veronica sect. Pseudolysimachium subsect. Spuriae (Holub) A. Jelen. 1977, Bull. Soc. Nat. Moscou, 82, 1: 152; Assejeva, 2002, Novit. Syst. P1. Vasc., 34: 162. - V. sect. Pseudolysimachium ser. Spuriae Boriss. 1955, F1. USSR, 22: 375, descr. ross.

Type: $V$. longifolia L.

1. V. longifolia L. s. str. 1753, Sp. P1. 1: 10; Bunge, 1829, in Ledeb., F1. Alt. 1: 26; Ledeb. 1847-1849, Fl. Ross. 3: 232; Krylov, 1939, F1. West. Sib. 10: 2438; Borisova, 1955, Fl. USSR, 22: 367; Gamajunova et Dmitrieva, 1965, Fl. Kazachst. 8: 67; Elenevsky, 1978, Sist. Geogr. Veronik SSSR Prilezh. Stran: 98; Polozhij, 1979, Fl. Krasnojarsk. kr. 9, 2: 12; Polozhij, 1996, Fl. Sib. 12: 32; Anenchonov et al., 2001, Key P1. Burjat.: 491; Wibe, 2001, Key P1. Kemerovsk. oblasti: 274; Assejeva, 2002, Novit. Syst. P1. Vasc., 34: 161; Kosachev, 2003, Key P1. Altaiskii krai: 356; Xiaoling, Nurbay, 2004, Fl. Xinjian. 4: 394; Krasnoborov, 2007, Key Pl. Republic Tuva: 411; Czepinoga et al., 2008, Konspekt fl. Irkutsk. oblasti: 214; Doronkin, 2012, Konspekt fl. Asiatskoi Rossii: 395. - V. luxurians Ledeb. 1829, Fl. Alt. 1: 27, in adnot. - Pseudolysimachion longifolium (L.) Opiz, 1852, Seznam: 80; Hong et Fisch. 1998, F1. China, 18: 68. - Veronica pseudolongifolia Printz, 1921, Veg. Siber.-Mongol. Front.: 380; Assejeva, 2002, Novit. Syst. P1. Vasc., 34: 161. - Veronica longifolia subsp. pseudolongifolia (Printz) Albach, 2008, Taxon, 53, 2: 4. Pseudolysimachion longifolium subsp. pseudolongifolium (Printz) Holub, 1998, Preslia, 70: 109. V. longifolia subsp. borealis (Trautv.) Kuvajev, 1981, Botan. Zeitschr. 66, 7: 953. - V. longifolia subsp. borealis (Trautv.) Assejeva, 2002, Novit. Syst. P1. Vasc., 34: 161, comb. superfl. - V. longifolia subsp. septentrionalis (Boriss.) Albach, 2008, Taxon, 53, 2: 4. - V. septentrionalis Boriss. 1955, Bot. Mater. Gerb. Bot. Inst. Komarova Akad. Nauk SSSR, 17: 341. - Pseudolysimachion septentrionale (Boriss.) Á. et D. Löve, 1976, Bot. Not. 128: 518. - P. longifolium subsp. septentrionale (Boriss.) Holub, 1967, in Holub et Pouzar, Folia Geobot. Phytotax. 2: 425.

Described from Europe (Habitat in Tataria, Austria, Suecia).

Lectotype (E. Fischer, 1997, Feddes Repert. 108: 114): Herb. Linn. No. 26.6 (LINN).

Moist forests or meadows but not dense birch or pine forests, creek margins.

Distribution in Siberia: West. Sib.: TJU - Jam (northern distribution margin - Obskaja Lippe, Nachodka bay), Tb, KU, OM, TO, NO, KE, AL - Ba,
Go; Central Sib.: KR - Ta (northernmost occurrence near Chatanga river), $\mathrm{Pu}, \mathrm{Tn}, \mathrm{Cha}, \mathrm{We}, \mathrm{TU}$; East. Sib.: IR - An, Pr, BU - Se, Juzh, Czi - Ka, Schi, JaK - Ol, Wi, Al, Jan, Ko.

General distribution: Scandinavia, Central to Western Europe, Northern Mediterranean, Caucasus, Russia (European part, Western and Eastern Siberia, Far East), Central Asia, Near East, Dschungarija-Kaschgarija, China, Japan, North America.

Chromosome number: $2 n=34$ (Fischer, 1969; Raitanen, 1967;), 64 (Polozhij, 1996), 68 (Meschkova, 1965; Probatova, Sokolovskaja, 1989; Raitanen, 1967), 90 (Polozhij, 1996).

Note 1. In the Altai and Sayan Mountains V.longifolia var. macrantha Boriss. is found with longovate leaves and glandular calyx.

Note 2 . The typical subspecies is certainly central for the evolution of the endemic $V$. sieboldiana Miq., V. subsessilis (Miq.) Carriere, V. ovata Nakai, that grow in Japan, Korea and the island of Tschetschzhudo.

Note 3. The main differences between $V$. maritima L. and $V$. longifolia L. are the calyx indumentum (simple long hairs in the former, short glandular hairs in the latter), the length of the bracts (longer or equal than pedicels in the former, or equal to shorter in the latter) and leaf base (cuneate to cordate in the former, rounded in the latter). Furthermore, leaves are often in whorls of 3-4 leaves in the European $V$. maritima and opposite in the Asian $V$. longifolia (Trávniček, 2000). The distinction is, however, questionable given the breadth of variation in the European part and the prevalence of hybridization.

Note 4. In LE one can find a possible hybrid $V$. longifolia L. $\times V$. daurica Stev. based on the determination of A. G. Elenevsky (Zabaikalsk. Area between the rivers Nercza and Kuenga, in the surroundings of the village Staraja Olova, meadows near the mouth of river Tungussky Olova. 09 VIII 1911. V. Sukatschev und G. Poplavskaja). This specimen requires further study.

2. V. taigischensis Stepanov, 1997, Botan. Zeitschr. 82, 9: 93; Doronkin, 2012, Konspekt fl. Asiatskoi Rossii: 396. - V. pseudolongifolia subsp. pseudolongifolia var. taigischensis (Stepanov) Assejeva, 2002, Novit. Syst. P1. Vasc., 34: 161. $V$. longifolia subsp. longifolia var. taigischensis (Stepanov) Assejeva, 2003, Botan. Zeitschr. 88, 1: 110. - Pseudolysimachion taigischense (Stepanov) Holub, 1998, Preslia, 70, 2: 109.

Described from West Sayan.

Type: Krasnojarskii krai, Karatuzkii district, River Tajgisch close to the mouth of river Kedran, 
on the steep bank of the river with moss, in Taiga (Pinus sibirica + Abies sibirica). 15 VI 1992. N. V. Stepanov (NS, iso - ALTB!, KGU, KRAS, LE!, MW).

In Taiga-forest.

Distribution in Siberia: Central Sib.: KR - We (known only from type locality).

General distribution: endemic.

Note: The species was found along the lower hills of the West Sayan near the village Tansybej (Stepanov, 1997).

3. V. sajanensis Printz, 1921, Contr. Fl. As. 3: 385; Borisova, 1955, Fl. USSR, 22: 375; Elenevsky, 1978, Sist. Geogr. Veronik SSSR Prilezh. Stran: 105; Polozhij, 1996, Fl. Sib. 12: 36; Anenchonov et al., 2001, Key P1. Burjat.: 491; Assejeva, 2002, Novit. Syst. P1. Vasc., 34: 161; Krasnoborov, 2007, Key P1. Republic Tuva: 411; Czepinoga et al., 2008, Konspekt fl. Irkutsk. oblasti: 214; Doronkin, 2012, Konspekt fl. Asiatskoi Rossii: 395.

Described from the Sayan Mountains (...Subalpine tracts in the Altaian).

Subalpine and alpine meadows, tundra in highalpine regions.

Distribution in Siberia: Central Sib.: KR - We, TU; East. Sib.: IR - An.

General distribution: endemic. Veronica sajanensis has once been recorded from Mongolia (Neuffer et al., 2003) but relevant record was based on the misidentified specimen of $V$. schmakovii (German et al., 2003).

Chromosome number: $2 n=18$ (Polozhij, 1996) likely misdetermined (Albach et al., 2004).

Note: The species takes on an intermediate position between section Longifoliae and Spicatae (Elenevsky, 1978), although an influence of section Pinnatae is possible based on DNA-sequence analysis (Kosachev, Mayland-Quellhorst, Albach, unpublished).

4. V. krasnoborovii Kosachev et Schaulo, 2013, Turczaninowia, 16, 3: 9.

Described from Sayan Mountains.

Type: Tuva ASSR, Ulug-Khem distr., Uyuk range, valley of Orto-Khem, right tributary of Bayan-Kol, alt. $1100 \mathrm{~m}$. Gravelly streamside. 02 VIII 1976. M. Lomonosova, A. Choodu (NS!, iso - NS!).

Gravel banks of river.

Distribution in Siberia: Central Sib.: TU.

General distribution: endemic.

5. V. spuria L. 1753, Sp. P1.: 10; Ledeb. 18471849, Fl. Ross. 3: 231, p. p.; Krylov, 1939, Fl. West. Sib. 10: 2440; Borisova, 1955, Fl. USSR, 22: 376;
Gamajunova et Dmitrieva, 1965, Fl. Kazachst. 8: 67; Elenevsky, 1978, Sist. Geogr. Veronik SSSR Prilezh. Stran: 105; Polozhij, 1996, Fl. Sib. 12: 38; Wibe, 2001, Key Pl. Kemerovsk. oblasti: 274; Assejeva, 2002, Novit. Syst. Pl. Vasc., 34: 162; Kosachev, 2003, Key P1. Altaiskii krai: 356; Xiaoling, Nurbay, 2004, Fl. Xinjian. 4: 394; Doronkin, 2012, Konspekt fl. Asiatskoi Rossii: 396. - V. paniculata L. 1759, Syst. Nat., ed. 10, 2: 849. - V. paniculata Pall. 1771, Reise, 1: 196; Bunge, 1829, in Ledeb. Fl. Alt. 1: 29. - Pseudolysimachion spurium (L.) Opiz, 1852, Seznam: 80. - Pseudolysimachion spurium (L.) Rauschert, 1966, Wiss. Z. Univ. Halle, cl. math.-natur. 15: 775; Trávniček, 2000, Preslia, 72: 431.

Described from Europe and Siberia (in australiore, Siberia).

Lectotype (E. Fischer, 1997, Feddes Repert. 108: 114): Herb. Linn. No. 26.56 (LINN).

Steppes, dry meadows, pine forests, birch forest margins.

Distribution in Siberia: West. Sib.: TJU $\mathrm{Tb}, \mathrm{KU}, \mathrm{OM}, \mathrm{NO}, \mathrm{AL}-\mathrm{Ba}$. Northern margin of distribution in Siberia is $56^{\circ} \mathrm{N}$ (Polozhij, 1996).

General distribution: Central Europe, Mediterranean, Caucasus, Russia (European part), Central Asia, Dschungarija-Kaschgarija.

Chromosome number: $2 n=34$ (Meschkova, 1965; Simonet, 1934).

6. V. linariifolia Pall. ex Link, 1820, in Sprengel, Schrader et Link, Jahrb. Gewächsk. 1, 3: 35; Borisova, 1955, Fl. USSR, 22: 386; Elenevsky, 1978, Sist. Geogr. Veronik SSSR Prilezh. Stran: 107; Polozhij, 1996, Fl. Sib. 12: 29; Anenchonov et al., 2001, Key P1. Burjat.: 491; Assejeva, 2002, Novit. Syst. P1. Vasc., 34: 163; Czepinoga et al., 2008, Konspekt fl. Irkutsk. oblasti: 214; Doronkin, 2012, Konspekt fl. Asiatskoi Rossii: 395. - V. cartilaginea Ledeb. 1829, Fl. Alt. 1: 28. - V. linariifolia subsp. cartilaginea (Ledeb.) Assejeva, 2002, Novit. Syst. Pl. Vasc., 34: 163. - V. rubicunda Ledeb. 1829, Fl. Alt. 1: 28. V. spuria $\beta$ Ledeb. 1847-1849, Fl. Ross. 3: 231. Pseudolysimachion linariifolium (Pall. ex Link) Yamazaki, 1968, Journ. Jap. Bot. 43, 10-11: 410.

Described from Transbaikalia.

Type: Herb. Willdenow Cat. No. 00167 (B).

Steppe, meadows, scree.

Distribution in Siberia: East. Sib.: IR - An, BU - Se, Juzh., Czi - Schi.

General distribution: Russia (Far East), Mongolia, China, Japan.

Chromosome number: $2 n=34$ (Albach et al., 2008; Marhold, 2008). 
Note: At Baikal Lake (Kultuk) and in the north of Mongolia one can find specimens with broadlanceolate leaves, dense indumentum of stem and petiole and with large pinkish flowers. This variation has been described in Russian only: $\boldsymbol{V}$. linariifolia var. baicalensis Boriss. (1955, Fl. USSR, 22: 389). Therefore, we here provide the Latin diagnosis and designate the type: Folia late lanceolata. Caulis et petioli foliis dense pubescentia. Corolla magna subroseus.

Type: At the river Irkut, Tunkinski district, Torski basin, near Krasny Jar Mountains, in willow shrub. 03 VII 1931. No. 27. V. I. Smirnov (IRK!, iso - IRK!).

Based on morphological characteristics, this species has similarities with $V$. spuria. Therefore, reports of $V$. spuria for the Flora of Mongolia may be wrong (see Kosachev, 2010; Smirnov et al., 2003).

2. Sect. Pseudolysimachium W. D. J. Koch, 1837, Syn. Fl. Germ.: 527. - Elenevsky, 1977, Bull. Soc. Nat. Moscou, 82, 1: 152; Elenevsky, 1978, Sist. Geogr. Veronik SSSR Prilezh. Stran: 110 (sub "Subsect. Pseudo-Lysimachium"). - Pseudolysimachion ser. Spicatae Holub, 1967, in Holub et Pouzar, Folia Geobot. Phytotax. 2, 4: 423. - Veronica sect. Pseudolysimachia ser. Spicatae Boriss. 1955, F1. USSR, 22: 380, descr. ross. $-V$. sect. Pseudolysimachia ser. Incanae Boriss., 1955, Fl. USSR, 22: 377, p. p., descr. ross. - V. sect. Pseudolysimachium ser. Spicatae Boriss. ex Yamazaki, 1957, Journ. Fac. Sci. Univ. Tokyo (Bot.), 7, 1-3: 139, p. p. - V sect. Pseudolysimachium subsect. Spicatae (Boriss. ex Yamazaki) Assejeva, 2002, Novit. Syst. P1. Vasc., 34: 162. - Pseudolysimachion sect. Pseudolysimachion: Trávniček, 1998, Preslia, 70, 3: 195.

Inflorescence thick, spike-like. Plants low with short rhizomes.

Type: lectotype of the subgenus.

7. V. incana L. 1753, Sp. Pl. 1: 10; Bunge, 1829, in Ledeb. Fl. Alt. 1: 32; Ledeb. 1847-1849, Fl. Ross. 3: 235; Krylov, 1939, Fl. West. Sib. 10: 2444; Borisova, 1955, Fl. USSR, 22: 377; Gamajunova et Dmitrieva, 1965, Fl. Kazachst. 8: 68; Elenevsky, 1978, Sist. Geogr. Veronik SSSR Prilezh. Stran: 110; Polozhij, 1979, Fl. Krasnojarsk. kr. 9, 2: 13; Polozhij, 1996, Fl. Sib. 12: 29; Anenchonov et al., 2001, Key Pl. Burjat.: 491; Wibe, 2001, Key Pl. Kemerovsk. oblasti: 274; Assejeva, 2002, Novit. Syst. P1. Vasc., 34: 163; Kosachev, 2003, Key P1. Altaiskii krai: 356; Krasnoborov, 2007, Key Pl. Republic Tuva: 410; Czepinoga et al., 2008, Konspekt fl. Irkutsk. oblasti: 214; Doronkin, 2012, Konspekt fl. Asiatskoi Rossii: 395. - V. bellidifolia Juz. 1950, Bot. Mater. Gerb. Bot. Inst. Komarova Akad. Nauk SSSR, 13: 297; Borisova, 1955, Fl. USSR, 22: 379.Pseudolysimachion incanum (L.) Holub, 1967, Folia Geobot. Phytotax. 2, 4: 424; Hong et Fischer, 1998, Fl. China, 18: 67.

Described from Ukraine.

Lectotype (Trávniček, 1998, Preslia, 70: 196): Herb. Linn. No. 26.7, second specimen from the left (LINN).

Steppe and rocky slopes, steppes, dry pine or lark forest.

Distribution in Siberia: West. Sib.: OM, NO, TO, KE, AL - Ba, Go; Central Sib.: KR - Tn (at the lower Tunguska river, Ilimpija, mouth of the lower Tschunka river), Cha, We, TU; East. Sib.: IR - An, BU -Se, JUZH, CZI - Schi, JAK - Ol, Wi, Al, Jan, Ko.

General distribution: Central Europe, Russia (European part, Western and Eastern Siberia), Far East, Central Asia, Mongolia, China.

Chromosome number: $2 n=32$ (Yakutia, Selerikan village - Polozhij, 1996), $2 n=2 x=34$ (Yakutia, Tscherski village - Polozhij, 1996), $2 n=4 x$ (based on flow cytometry: Kosachev, Mayland-Quellhorst, Albach, unpublished).

Note: The species is tetraploid in Western Europe (Albach et al., 2008). In the Asiatic part of Russia and even in the south of Siberia diploid and tetraploid plants can be found, but only diploids in Yakutia. Further studies of karyology and DNA are necessary. Counts of 32 chromosomes are likely misdeterminations (chromosomes in the subgenus are small and sticky, which makes exact determination difficult - Albach et al., 2008).

8. V. sergievskiana Polozhij, 1996, Fl. Sib. 12: 37; Assejeva, 2002, Novit. Syst. Pl. Vasc., 34: 163; Doronkin, 2012, Konspekt fl. Asiatskoi Rossii: 395.

Described from East Siberia.

Type: Tschitaer area, on the banks of the Nertscha river, below the village Zjulzja, $52^{\circ} 33^{\prime} \mathrm{N}, 116^{\circ} 12^{\prime} \mathrm{E}$. In the steppe. 04 VII 1952. L. P. Sergijevskaja, L. I. Obolenzev (TK).

Steppe.

Distribution in Siberia: East. Sib.: Czi - Schi (type locality-Zjulzja village; Schischkino village).

General distribution: endemic.

Chromosome number: $2 n=$ ?

Note: The species is little known and requires further study.

9.1. V. spicata L. s. str. 1753, Sp. P1.: 10; Bunge, 1829, in Ledeb. Fl. Alt. 1: 30, p. p.; Ledeb. 1847- 
1849, Fl. Ross. 3: 233, p. p.; Krylov, 1939, Fl. West. Sib. 10: 2442; Borisova, 1955, Fl. USSR, 22: 381, p. p.; Gamajunova et Dmitrieva, 1965, Fl. Kazachst. 8: 68; Elenevsky, 1978, Sist. Geogr. Veronik SSSR Prilezh. Stran: 112; Polozhij, 1996, Fl. Sib. 12: 38; Wibe, 2001, Key P1. Kemerovsk. oblasti: 276; Assejeva, 2002, Novit. Syst. P1. Vasc., 34: 163; Kosachev, 2003, Key Pl. Altaiskii krai: 357; Czepinoga et al., 2008, Konspekt fl. Irkutsk. oblasti: 214; Doronkin, 2012, Konspekt fl. Asiatskoi Rossii: 395. V. psilophylla Nevski ex Kryl. 1939, Fl. West. Sib. 10: 2442. - Pseudolysimachion spicatum (L.) Opiz, 1852, Seznam: 80; Hong et Fischer, 1998, Fl. China, 18: 68 .

Described from Europe.

Lectotype (E. Fischer, 1997, Feddes Repert. 108: 114): Herb. Linn. No. 26.10 (LINN).

Steppe, dry meadows, rocky slopes.

Distribution in Siberia: West. Sib.: TJU - Tb, KU, OM, TO, NO, KE, AL - Ba; Central Sib.: KR Cha; East. Sib.: IR - An.

General distribution: Scandinavia, Central and Western Europe, Mediterranean, Caucasus, Russia (European part, West and East Siberia), Central Asia (North), North America (introduced).

Chromosome number: $2 n=34$ (Fischer, 1969; Polozhij, 1996), 68 (see Albach et al., 2008).

9.2. V. spicata subsp. kamelinii Kosachev, 2003, Turczaninowia, 6, 1: 19; Doronkin, 2012, Konspekt fl. Asiatskoi Rossii: 395.

Described from the Altai.

Type: Altai krai, Kurjinskii district, Kolywanskii ridge, northwestern slope of Mount Sinjucha near Kolyvanstroja, $51^{\circ} 15.5^{\prime} \mathrm{N}, 82^{\circ} 34^{\prime}$ ' E. AA No. 272. 18 VI 1999. Kamelin R. V., Schmakov A. I., Tschubarov I., Kostjukov S., Antonjuk E., Kosachev P. (ALTB).

Mixed forest.

Distribution in Siberia: West. Sib.: AL - Ba (type locality).

General distribution: endemic.

Chromosome number: $2 n=2 x$ (= ca. 34) (our data based on flow cytometry, Kosachev, Albach, Mayland-Quellhorst, unpublished).

Note: Nuclear DNA (ITS) from this subspecies contains ribotypes characteristic of $V$. spicata from Bashkiria and $V$. orchidea Crantz (Kosachev, Mayland-Quellhorst, Albach, unpublished).

9.3. V. spicata subsp. paczoskiana (Klok.) Kosachev, 2003, Turczaninowia, 6, 1: 22. - V. paczoskiana Klok. 1976, Novosti sistematiki vyssich i nizsich rastenij (Kiev) 1975: 105; Doronkin, 2012,
Konspekt fl. Asiatskoi Rossii: 395. - V. spicata subsp. spicata var. pseudoorchidea Pacz. 1909, Zapiski Novorossijskago Obscestva Estestvoispytatelej 34: 144; Assejeva, 2002, Novit. Syst. Pl. Vasc., 163. - V. pseudorchidea (Pacz.) Klok. 1976, Novosti sistematiki vyssich i nizsich rastenij (Kiev) 1975: 109, quoad nomen. - V. psilophylla Nevski ex Kryl. 1939, Fl. West. Sib. 10: 2442, nom. nud. - V. spicata subsp. spicata auct., non L.: Tzvelev, 1981, Bull. Soc. Nat. Moscou, 86, 6: 85.

Described from Ukraine.

Type: Ukraine, Tscherkassk province, Kanevskii district, Michajlovka village, Michajlovskii pine forest (pinetum pteridiosum), 27 VII 1972. M. Klokov (Kiev) (KW).

Pine forest.

Distribution in Siberia: West. Sib.: KU, TJU $\mathrm{Tb}, \mathrm{OM}, \mathrm{NO}, \mathrm{TO}, \mathrm{AL}-\mathrm{Ba}$, Go (village Tschemal; river Tschulyschman).

General distribution: Ukraine, Belarus.

Chromosome number: $2 n=2 x=34$ (Androschtschuk, 1988; our data based on flow cytometry, Kosachev, Albach, Mayland-Quellhorst, unpublished)).

Note: As demonstrated by recent study (Kosachev, Ebel, 2010), the subspecies is distributed in pine forests of West Siberia. It may be a more ancestral form in $V$. spicata.

10. V. porphyriana Pavl. 1951, Vestnik Akad. Nauk KazSSR, 4: 92; Borisova, 1955, Fl. USSR, 22: 382; Sergievskaja, 1964, Fl. West. Sib. 12, 2: 3447; Gamajunova et Dmitrieva, 1965, Fl. Kasachst. 8: 69; Polozhij, 1979, Fl. Krasnojarsk. kr. 9, 2: 14; Polozhij, 1996, Fl. Sib. 12: 33; Wibe, 2001, Key Pl. Kemerovsk. oblasti: 275; Assejeva, 2002, Novit. Syst. P1. Vasc., 34: 164; Kosachev, 2003, Key Pl. Altaiskii krai: 357; Krasnoborov, 2007, Key Pl. Republic Tuva: 413; Doronkin, 2012, Konspekt fl. Asiatskoi Rossii: 395. - V. spicata L. var. viscosissima Kar. et Kir. 1841, Bull. Soc. Nat. Mosc. [14], 4: 721 (No 664). - V. spicata subsp. porphyriana (Pavl.) A. Jelen. 1978, Sist. Geogr. Veronik SSSR Prilezh. Stran: 115. - V. spicata auct., non L.: Xiaoling, Nurbay, 2004, Fl. Xinjian. 4: 393. - Pseudolysimachion porphyrianum (Pavl.) Holub, 1967, Folia Geobot. Phytotax. (Praha), 4, 2: 425. - P. spicatum auct., non Opiz: Hong et al., 1998, Fl. China, 18: 68, p. p.

Described from Transili Alatau.

Type: Kazakhstan, prov. Almaatensis, montes Alatau Transiliensis, ad fontes fl. Talgar Sinistrum in cobresietis, $3000 \mathrm{~m}$ alt., No. 351, 27 VII 1940, fl. et fr. immat., leg., P. P. Poljakov" (AA!). 
In the alpine and subalpine zone of mountains, meadows, steppic and rocky slopes, fir- (Tian Shan) and pine-forest, rocky and steppic river valleys in the foothills.

Distribution in Siberia: West. Sib.: KE, AL - Ba, Go; Central Sib.: KR - Cha, We, TU.

General distribution: Southern Central Asia.

Chromosome number: $2 n=2 x=34$ (Afanasjeva, Meschkova, 1961; Rostovtseva, Krasnoborov, Krasnikova, 1981, sub V. spicata; our data based on flow cytometry, Kosachev, Mayland-Quellhorst, Albach, unpublished).

Note: This species from the mountainous region, growing from foothills to high alpine zone is according to our research a central taxon in the subgenus that is involved in hybridization with several other taxa.

11. V. $\times$ czemalensis Kosachev et Albach, 2013, Turczaninowia, 16, 3: 12.

Described from the Altai.

Type (with isotypes): “Tuva Republic, Ovyurskii distr., Tzagan-Shibetu range, upper reaches of Arzaiti, left bank. $50^{\circ} 27^{\prime} 40.3^{\prime \prime}$ N, 9049'49.4” E, KZ 2104. 16 VII 2006. A. I. Shmakov, S. V. Smirnov, M. G. Kutsev, A. V. Vaganov, R. A. Zubov, M. S. Ivanova, A. A. Kechaikin, A. P. Shalimov" (ALTB).

Meadows, steppes and scrubs in lower mountains of the Northern Altai, high mountain steppes in the Zagan-Schibetu Mountains.

Distribution in Siberia: West. Sib.: AL - Go; Central Sib.: TU.

General distribution: endemic.

Note: This taxon has been found in the process of molecular-genetic studies (Kosachev, MaylandQuellhorst, Albach, unpublished). The hybrid requires further study in the zone of sympatry of $V$. porphyriana and $V$. incana.

3. Sect. Pinnatae (Holub) Kosachev et Albach, stat. nov. - Pseudolysimachion ser. Pinnatae Holub, 1967, in Holub et Pouzar, Folia Geobot. Phytotaxon. 2, 4: 423. - Veronica subsect. Pinnatae (Holub) Jelen. 1977, Bull. Soc. Nat. Moscou, 82, 1: 152; Assejeva, 2002, Novit. Syst. P1. Vasc., 34: 162. - Veronica sect. Pseudolysimachia ser. Pinnatae Boriss. 1955, Fl. USSR, 22: 368, p. p. (descr. ross.).

Leaves pinnatifid or pinnatipartite.

Type: V. pinnata L.

12. V. reverdattoi Krasnob. 1973, Novosti Geogr. Sist. Rast. Sibiri 1973: 4; Polozhij, 1979, Fl. Krasnojarsk. kr. 9, 2: 14; Polozhij, 1996, Fl. Sib. 12:
36; Assejeva, 2002, Novit. Syst. Pl. Vasc., 34: 162; Krasnoborov, 2007, Key Pl. Republic Tuva: 410; Doronkin, 2012, Konspekt fl. Asiatskoi Rossii: 395. Described from Khakassia.

Type: Krasnojarskii krai, Khakassia autonomous region, Taschtypski district, in the vicinity of the village Karagajskaja, $2 \mathrm{~km}$ north of it, stony steppe. 14 VIII 1970, non-flowering, I. M. Krasnoborov (NS, iso - LE! ALTB!).

Stony slopes, various types of steppe.

Distribution in Siberia: Central Sib.: KR - Cha, TU (Teeli village; Lake Kara-Chol).

General distribution: endemic.

Chromosome number: $2 n=$ ?

Note: I. M. Krasnoborov (1973) noted the similarity of the species with $V$. sessiliflora Bunge (probably a hybrid of $V$. pinnata and $V$. porphyriana Kamelin, 1998), but our analysis of cpDNA of the type material revealed similarity to $V$. incana (Kosachev, Mayland-Quellhorst, Albach., unpublished).

13.1. V. pinnata L. s. str. 1767, Mantissa, 1: 24; Bunge, 1829, in Ledeb. Fl. Alt. 1: 33; Ledeb. 18471849, Fl. Ross. 3: 230; Krylov, 1939, Fl. West. Sib. 10: 2446; Borisova, 1955, Fl. USSR, 22: 391; Gamajunova et Dmitrieva, 1965, Fl. Kazachst. 8: 73; Elenevsky, 1978, Sist. Geogr. Veronik SSSR Prilezh. Stran: 118; Polozhij, 1979, Fl. Krasnojarsk. kr. 9, 2: 14; Polozhij, 1996, Fl. Sib. 12: 32; Wibe, 2001, Key Pl. Kemerovsk. oblasti: 274; Assejeva, 2002, Novit. Syst. Pl. Vasc., 34: 162; Kosachev, 2003, Key P1. Altaiskii krai: 355; Krasnoborov, 2007, Key Pl. Republic Tuva: 410; Czepinoga et al., 2008, Konspekt fl. Irkutsk. oblasti: 214; Doronkin, 2012, Konspekt fl. Asiatskoi Rossii: 395. - Pseudolysimachion pinnatum (L.) Holub, 1967, Folia Geobot. Phytotax. 2, 4: 428; Hong et Fischer, 1998, Fl. China, 18: 67.

Described from Siberia (Habitat in Siberia. Laxman).

Neotype (Elenevsky, 1978, 1. c.: 118, as "type"): Herb. Linn. 26.18 (LINN).

Steppes of lower foothills and mountains, rocky and gravelly banks of rivers.

Distribution in Siberia: West. Sib.: KE, AL - Ba, Go; Central Sib.: KR - Cha, We, TU; East. Sib.: IR - An.

General distribution: Central Asia, China, Mongolia (northern and central parts).

Chromosome number: $2 n=2 x$ (= ca. 34 ) (our data based on flow cytometry, Kosachev, MaylandQuellhorst, Albach, unpublished).

13.2. V. pinnata subsp. nana (Kryl.) Polozhij, 1996, Fl. Sib. 12: 33; Assejeva, 2002, Novit. Syst. 
P1. Vasc., 34: 162; Doronkin, 2012, Konspekt fl. Asiatskoi Rossii: 395. - V. pinnata f. nana Kryl. 1907, Fl. Alt.: 939; Krylov, 1939, Fl. West. Sib. 10: 2447.

Described from Altai.

Type: "Altaj austro-orientalis, in fluxu Czegan Usun et Elangasch. 01 VII 1901. P. Krylov" (TK!).

Dry, rocky slopes, mountain steppe.

Distribution in Siberia: West. Sib: AL - Go;

Central Sib.: TU.

General distribution: Mongolia.

Chromosome number: $2 n=2 x$ (= ca. 34) (our data based on flow cytometry, Kosachev, MaylandQuellhorst, Albach, unpublished).

Note: Based on the study of nuclear ITS it is clear that $V$. pinnata subsp. nana contains exclusively pinnata-ribotypes and $V$. pinnata subsp. pinnata contains a mixture of pinnata-ribotypes with spicata- or longifolia-ribotypes (Kosachev, Mayland-Quellhorst, Albach, unpublished).

4. Sect. Dauricae (Holub) Kosachev et Albach, stat. nov. - Pseudolysimachion ser. Dahuricae Holub, 1967, Folia Geobot. Phytotax. 2, 4: 422. Veronica subsect. Dauricae (Holub) A. Jelen. 1977, Bull. Soc. Nat. Moscou, 82, 1: 152. - V. sect. Pseudolysimachia ser. Grandes Boriss. 1955, Fl. USSR, 22: 371, p. p. (descr. ross.).

Leaves crenate to pinnatifid. Inflorescences thick. Flowers white.

Type: $V$. daurica Stev.

14. V. daurica Stev. s. str. 1817, Mem. Soc. Nat. Mosc. 5: 339; Borisova, 1955, Fl. USSR, 22: 372; Elenevsky, 1978, Sist. Geogr. Veronik SSSR Prilezh. Stran: 108; Polozhij, 1996, Fl. Sib. 12: 28; Anenchonov et al., 2001, Key P1. Burjat.: 491, "dahurica"; Assejeva, 2002, Novit. Syst. Pl. Vasc., 34: 161; Doronkin, 2012, Konspekt fl. Asiatskoi Rossii: 394. - Veronica grandis Fisch. ex Spreng. 1821, Neue Entdeck. 2: 122. - Pseudolysimachion dahuricum (Stev.) Yamazaki, 1968, Journ. Jap. Bot. 47, 1-3: 410 .

Described from Dauria.

Type: In Dauria circa Doroninsk (LE!).

Steppe, meadows, mixed and poplar forest, sandy-stony slopes.

Distribution in Siberia: East. Sib.: BU - Se, JUZH, CZI - Schi.

General distribution: Russia (der Far East), Mongolia, China, Japan.

Chromosome number: $2 n=32,34$ (Albach et al., 2008; Graze, 1935).
Note: One of the most plesiomorphic types of the subgenus. According to morphological characteristics and genetic similarities, the species is close to $V$. schmidtiana and $V$. nakaiana Ohwi, which are sister to the rest of the subgenus (Kosachev, Mayland-Quellhorst, Albach, unpublished).

Intersectional species

15. $\boldsymbol{V} \times$ altaica Kosachev, 2003, Turczaninowia, 6, 1: 26; Doronkin, 2012, Konspekt fl. Asiatskoi Rossii: 394. $-V$. $\times$ austrosibirica Kosachev, 2003, Turczaninowia, 6, 1: 28; Doronkin, 2012, Konspekt fl. Asiatskoi Rossii: 394.

Described from West Altai.

Type: Altai krai, Tretjakovskii district, Porutschikova Mountains, $12 \mathrm{~km}$ south of Ekaterininskoe village, $50^{\circ} 46.5^{\prime} \mathrm{N}, 82^{\circ} 04^{\prime} \mathrm{E}$. TASCH No. 692,03 VII 1996. Kamelin R. V., Shmakov A. I., Terechina T. A., Smirnov S., Solovjev A., Tikhonov D., German D., Antonjuk E. (ALTB).

Foothill steppe, rocky slopes and scree of the mountains.

Distribution in Siberia: West. Sib.: AL - Ba, Go. General distribution: endemic.

Chromosome number: $2 n=2 x$ (= ca. 34 ) (our data based on flow cytometry, Kosachev, MaylandQuellhorst, Albach unpublished).

Note: contains nuclear DNA ribotypes characteristic of $V$. spicata subsp. kamelinii and $V$. pinnata L., confirming a hybridogeneous origin of the species (Kosachev, Mayland-Quellhorst, Albach, unpublished).

16. $\boldsymbol{V} \times$. sessiliflora Bunge (pro sp.), 1829, in Ledeb., Fl. Alt. 1: 32; Ledeb. 1829, Ic. pl. Fl. Ross., t. 126; Ledeb. 1847-1849, Fl. Ross. 3: 230; Krylov, 1939, Fl. West. Sib. 10: 2447; Borisova, 1955, Fl. USSR, 22: 391; Gamajunova et Dmitrieva, 1965, Fl. Kazachst. 8: 72; Polozhij, 1996, Fl. Sib. 12: 37; Assejeva, 2002, Novit. Syst. P1. Vasc., 34: 162; Krasnoborov, 2007, Key Pl. Republic Tuva: 410; Doronkin, 2012, Konspekt fl. Asiatskoi Rossii: 395.V. pinnata var. sessiliflora (Bunge) Härle, 1932, Bibl. Bot. 104: 30.

Described from the Central Altai: "Hab. in locis sterilibus deserti ad fluvios Kurai et Tschuja (B.)".

Lectotype (Kosachev, 2011, Novit. Syst. P1. Vasc. 43: 136): [27 Altai] Veronica sessiliflora Bunge ap[ud] Ledeb. (- pinnatifida Bge in litt.). In deserto Kuraico legit Dr. Bunge, Julio 1826. Herb. C. A. Mey[er] (LE!).

Isolectotype: Herb. Ledebour Altai, 1826 (LE!). Mountain steppe, desert steppes along rivers. 
Distribution in Siberia: West. Sib.: AL - Go; Central Sib.: TU.

General distribution: endemic.

Chromosome number: $2 n=2 x$ (= ca. 34) (our data based on flow cytometry, Kosachev, MaylandQuellhorst, Albach, unpublished).

Note: Based on our analysis of cpDNA, the species is similar to $V$. incana and $V$. porphyriana (Kosachev, Mayland-Quellhorst, Albach, unpublished). A relationship with $V$. pinnata, however, has not been demonstrated yet.

17. V. $\times$ schmakovii Kosachev, 2003, Turczaninowia, 6, 1: 16; Kosachev, 2010, Turczaninowia, 13, 1: 50; Doronkin, 2012, Konspekt fl. Asiatskoi Rossii: 395.

Described from Altai.

Type: Republic of Altai, Kosch-Agatschskii district, Ukok plateau, lower stretch of river Kara-Bulak close to watch tower Ak-Alacha, $49^{\circ} 16^{\prime}, 8^{\circ} 28^{\prime}$, AJUK No. 3321, 28 VII 1998. Kamelin R. V., Shmakov A. I., Smirnov S., Kosachev P., Tikhonov D., Antonjuk E. (ALTB).

Creek margins, dwarf birch scrub, forest margin, especially lark forest in high alpine regions.

Distribution in Siberia: West. Sib.: AL - Go; Central Sib.: TU.

General distribution: Western Mongolia. It may stay in China (Xinjiang).

Chromosome number: $2 n=2 x$ (= ca. 34) (our data based on flow cytometry, Kosachev, MaylandQuellhorst, Albach, unpublished).

Note: based on molecular phylogenetic analysis in the subgenus (Kosachev, Mayland-Quellhorst, Albach, unpublished) the species is related to
$V$. longifolia (AFLP) and V. porphyriana (cpDNA), confirming the hybrid origin of $V . \times$ schmakovii.

18. V. $\times$ grisea Kosachev et A. L. Ebel, 2010, Sist. Zametki Mater. Gerb. Krylova 102: 9; Doronkin, 2012, Konspekt fl. Asiatskoi Rossii: 395. - Veronica neglecta Vahl, 1804, Enum. Pl. 1: 59, non F. W. Schmidt, 1795, Fl. Boem. 1: 12.

Described from Siberia.

Type: Herb. Copenhagen. No.78/11 (C).

Meadows.

Distribution in Siberia: West. Sib.: KU?, KR Cha (Schirinskii district, in the vicinity of Efremkino village).

General distribution: Eastern Europe, Russia (southern part of European part).

Note: Based on the analysis of cpDNA, a close relationship with $V$. incana is confirmed (Kosachev, Mayland-Quellhorst, Albach, unpublished).

19. $\boldsymbol{V} \times$ kolyvanensis Kosachev et Shmakov, 2013, Turczaninowia, 16, 3: 11.

Described from western Altai.

Type (with isotypes): Altai province, Kuryinskiy distr., valley of Loktevka in $7 \mathrm{~km}$ downstream of Kolyvan, $51^{\circ} 17,5^{\prime} \mathrm{N}, 82^{\circ} 29^{\prime} \mathrm{E}, \mathrm{KOL} 1957.22 \mathrm{VI}$ 2003. A. I. Shmakov, S. V. Smirnov, M. G. Kutzev, I. V. Naumov, A. V. Vaganov (ALTB).

Shrubs.

Distribution in Siberia: West. Sib.: AL - Ba.

General distribution: endemic.

Note: Likely a hybrid of V. spuria and V. spicata.

This study has been conducted with financial support by RFFI (No. 13-04-90786) and DAAD (No. 10.33.2011 and No. 11.9175.2014).

\section{LITERATURE}

Afanas'eva N. G., Meshkova L. Z. Application of kariogeographical researches to philogeny genus Veronica (Russia) //Bot. Journ. [Bot. Zhurn.], 1961. - Vol. 46, No. 2. - P. 247-258 [In Russian]. (Афанасьева Н. Г., Мешкова Л. 3. Применение кариогеографических исследований к филогении рода Veronica // Бот. журн., 1961. - Т. 46, № 2. - C. 247-258).

Albach D. Further arguments for the rejection of paraphyletic taxa: Veronica subgen. Pseudolysimachium (Plantaginaceae) // Taxon, 2008. - Vol. 57(1). - P. 1-6.

Albach D., Chase M. Incongruence in Veroniceae (Plantaginaceae): evidence from two plastid and a nuclear ribosomal DNA region // Molecular Phylogenetics and Evolution, 2004. - Vol. 32. - P. 183-197.

Albach D., Fischer M. AFLP- and genome size analyses: contribution to the taxonomy of Veronica subg. Pseudolysimachium sect. Pseudolysimachion (Plantaginaceae), with a key to the European taxa // Phytologia Balcanica, 2003. - Vol. 9(3). - P. 401-424.

Albach D., Martinez-Ortega M., Delgado L., Weiss-Schneeweiss H., Özgökce F., Fischer M. Chromosome numbers in Veroniceae (Plantaginaceae): Review and several new counts // Ann. Missouri Bot. Gard., 2008. - Vol. 95. - P. 543-566.

Albach D., Martinez-Ortega M., Fischer M., Chase M. A new classification of the tribe Veroniceae - problems and a possible solution // Taxon, 2004. - Vol. 53(2). - P. 429-452. 
Androschuk A. F. Numbers of chromosomes of some species of Veronica (Scrophulariaceae) in Ukraine (Russia) // Bot. Journ. [Bot. Zhurn.], 1988. - Vol. 73, No. 3. - P. 454-455 [In Russian]. (Андроцук А. Ф. Числа хромосом некоторых видов рода Veronica (Scrophulariaceae) на Украине // Бот. журн., 1988. - Т. 73, № 3. - C. 454-455).

Angiosperm Phylogeny. Group II. An update of the Angiosperm Phylogeny Group classification for the orders and families of flowering plants: APG II // Botanical Journal of the Linnean Society, 2003. - Vol. 141(2). - P. $399-436$.

Angiosperm Phylogeny. Group III. An update of the Angiosperm Phylogeny Group classification for the orders and families of flowering plants: APG III // Botanical Journal of the Linnean Society, 2009. - Vol. 161(2). - P. $105-121$.

Assejeva L. A. System of genus Veronica L. (Scrophulariaceae) floras of Russia (Russia) // Novit. Syst. Vasc. Pl. [Novosti Sist. Vyssh. Rast.], 2002. - Vol. 34. - P. 159-173 [In Russian]. (Асеева Л. А. Система рода Veronica L. (Scrophulariaceae) флоры России // Новости сист. высш. раст., 2002. - Т. 34. - С. 159-173).

Borisova A. G. Veronica L. // Flora of USSR. Vol. 22. - Moscow and Leningrad: Publishers of Academy of Sciences of USSR, 1955. - P. 329-500 [In Russian]. (Борисова А. Г. Вероника - Veronica L. // Флора СССР. Т. 22. М.-Л.: Изд-во АН СССР, 1955. - С. 329-500).

Chromosomal numbers of flowering plants / Ed. An. A. Fedorov [Pod red. An. A. Fedorova]. - Leningrad: Nauka, 1969. - 926 p. [In Russian] (Хромосомные числа цветковых растений / Под ред. Ан. А. Федорова. - Л.: Наука, 1969. - 926 c.).

Elenevsky A. G. Notes about Veronica of section Pseudolysimachia C. Koch (Russia) // Biol. sciences [Biol. Nauki], 1968. - No. 11. - P. 65-69 [In Russian]. (Еленевский А. Г. Заметки о верониках секции Pseudolysimachia С. Koch // Биол. науки, 1968. - № 11. - С. 65-69).

Elenevsky A. G. To systematics of Veronica spicata L. s. 1. (Russia) // Novit. Syst. Vasc. Pl. [Novosti Sist. Vyssh. Rast.], 1971. - Vol. 8. - P. 215-227 [In Russian]. (Еленевский А. Г. К систематике Veronica spicata L. s. 1. // Новости сист. высш. раст., 1971. - Т. 8. - С. 215-227).

Elenevsky A. G. System of genus Veronica L. (Russia) // Byull. MOIP. Otd. biol., 1977. - Vol. 82, iss. 1. - P. 149-160 [In Russian]. (Еленевский A. Г. Система рода Veronica L. // Бюлл. МОИП, отд. биол., 1977. - Т. 82, вып. 1. - C. 149-160).

Elenevsky A. G. Systematics and geography of Veronica of the USSR and the adjacent countries [Sistematika i geographia veronik SSSR i prilezhaschich stran]. - Moscow: Nauka, 1978. - 259 p. [In Russian]. (Еленевский А. Г. Систематика и география вероник СССР и прилежащих стран. - М.: Наука, 1978. - 259 с).

Fischer M. Einige Chromosomenzahlen aus den Gattungen Veronica, Pseudolysimachion, Paederota, Wulfenia und Lagotis (Scrophulariaceae) // Österr. bot. Zeit., 1969. - Vol. 116, iss. 1-5. - P. 430-443.

German D. A., Neuffer B., Friesen N., Hurka H. Contribution to the knowledge of the flora of the Mongolian Altai II // Feddes Repert., 2003. - Vol. 114. iss. 7-8. - P. 632-637 [doi: 10.1002/fedr.200311018].

Graze H. Weitere Chromosomenuntersuchungen bei Veronica Arten der Sektion Pseudolysimachia // Jahrb. Wiss. Bot. 81, 1935. - P. 609-662.

Kamelin R. $\boldsymbol{V}$. Proceedings on stories of flora of Asia (the Altay highland)]. - Barnaul: Publishing of the Altay state university, 1998. - 240 p. [In Russian]. (Камелин P. В. Материалы по истории флоры Азии (Алтайская горная страна). - Барнаул, 1998. - 240 с).

Klokov M. V. About Veronica spicata group // Novit. Syst. Pl. non Vasc. and Vasc. (1975) [Novosti Sist. Nizsh. i Vyssh. Rast. (1975)], 1976. - P. 92-111 [In Russian]. (Клоков М. В. О верониках колосистых // Новости сист. высш. и низш. раст. (1975), 1976. - C. 92-111).

Kosachev P. A. Review of the section Pseudolysimachium W. D. J. Koch (Genus Veronica L., Scrophulariaceae) in Altai Mountains // Turczaninowia, 2003. - Vol. 6, iss. 1. - C. 11-33 [In Russian]. (Косачев П. А. Обзор секции Pseudolysimachium W. D. J. Koch рода Veronica L. (Scrophulariaceae) во флоре Алтайской горной страны // Turczaninowia, 2003. - Т. 6, вып. 1. - C. 11-33).

Kosachev P. A. Synops is of the families Scrophulariaceae Juss. and Pediculariaceae Juss. of Altai Mountain Country // Turczaninowia, 2010. - Vol. 13, iss. 1. - C. 19-102 [In Russian]. (Косачев П. А. Конспект сем. Scrophulariaceae Juss. и Pediculariaceae Juss. Алтайской горной страны // Turczaninowia, 2010. - Т. 13, вып. 1. - C. 19-102).

Kosachev P. A. Typification of names of some species of Veronica L. and Linaria Mill. (Plantaginaceae) described from Siberia (in Russian) // Novit. Syst. Vasc. Pl. [Novosti Sist. Vyssh. Rast.], 2011. - Vol. 43. - P. 132-143 [In Russian]. (Косачев П. A. Типификация названий некоторых видов Veronica L. и Linaria Mill. (Plantaginaceae), описанных из Сибири // Новости сист. высш. раст., 2011. - Т. 43. - С. 132-143.

Kosachev P. A., Ebel A. L. Notes on Veronica L. in Siberia // Animadversiones Systematicae ex Herbario Kryloviano Universitatis Tomskensis [Sistematiczeskiye zametki po materialam Gerbarija im. P. N. Krylova Tomskogo Universiteta], 2010. - Iss. 102. - Р. 3-11 [In Russian]. (Косачев П. А., Эбель А. Л. Сообщение о верониках Сибири // Сист. зам. Герб. им. П.Н. Крылова Томск. ун-та, 2010. - Вып. 102. - С. 3-11).

Kosachev P. A., German D. A. New species of genus Veronica L. (Scrophulariaceae) from the Western Mongolia // Novit. Syst. Vasc. P1. [Novosti Sist. Vysch. Rast.], 2004. - Vol. 36. - P. 209-212 [In Russian]. (Косачев П. А., Герман Д. А. Новый вид рода Veronica L. (Scrophulariaceae) из Западной Монголии // Новости сист. высш. раст., 2004. - Вып. 36. - С. 209-212). 
Krasnoborov I. M. New species of genus Veronica from Krasnoyarskii krai // Novit. Geogr. and Syst. Pl. Siberia [Nov. geogr. i sist. rast. Sibiri], 1973. - P. 4 [In Russian]. (Красноборов И. М. Новый вид рода Vегопіса из Красноярского края // Нов. геогр. и сист. раст. Сибири, 1973. - С. 4).

Malyshev L. I. Preface // Flora of Siberia. T. 1. Lycopodiaceae - Hydrocharitaceae. - Novosibirsk: Nauka, sib. otd-nie, 1988. - P. 5-11 [In Russian]. (Малышев Л. И. Предисловие // Флора Сибири. Т. 1. Lycopodiaceae - Нydrocharitaceae. - Новосибирск: Наука, Сиб. отд-ние, 1988. - С. 5-11).

Manukjan L. K. Palinosystematics of genus Veronica L. // Biol. Journ. of Armenia [Biol. zhurn. Armenii], 1984. Vol. 37, iss. 7. - P. 548-556 [In Russian]. (Манукян Л. К. Палиносистематика рода Veronica L. // Биол. журн. Армении, 1984. - Т. 37, вып. 7. - С. 548-556).

Meshkova L. Z. Application of karyological researches to systematics of genus Veronica L. - Avtoref. dis.... kand. biol. nauk. - Kazan, 1965. - 16 p. [In Russian]. (Мешкова Л. З. Применение кариологических исследований к систематике рода Veronica L.: Автореф. дис.... канд. биол. наук. - Казань, 1965. - 16 с.).

Neuffer B., Oyuntsetseg B., Schamsran Z., Friesen N., Hurka H. Contribution to the knowledge of the flora of the Mongolian Altai // Feddes Repert., 2003. - Vol. 114. iss. 5-6. - P. 358-371 [doi: 10.1002/fedr.200311004].

Polozhij A. V. Veronica L. // Flora of Siberia. Vol. 12: Solanaceae - Lobeliaceae. - Novosibirsk, 1996. - P. 26-47 [In Russian]. (Положий А. В. Veronica L. - Вероника // Флора Сибири. Т. 12: Solanaceae - Lobeliaceae. - Hoвосибирск, 1996. - С. 26-47).

Probatova N. S., Kazanovsky S. G., Rudyka E. G., Seledets V. P., Ovchinnikova S. V. IAPT/IOPB chromosome data 16 (ed. Marhold) // Taxon, 2013. - Vol. 62(6). - P. 1359-1360.

Probatova N. S., Sokolovskaja A. P., Rudyka E. G. Chromosome numbers of some species of vascular plants of island Kunashir, Kuriles // Bot. Journ. [Bot. Zhurn.], 1989. - Vol. 74, No. 11. - P. 1675-1678 [In Russian]. (Пробатова Н. С., Соколовская А. П., Рудыка Э. Г. Числа хромосом некоторых видов сосудистых растений о-ва Кунашир, Курильские острова // Бот. журн., 1989. - Т. 74, № 11. - С. 1675-1678).

Raitanen P. R. Taxonomic studies on Veronica longifolia and V. spicata in Eastern Fennoscandia // Ann. Bot. Fenn., Helsinki, 1967. - Vol. 4. - P. 471-485.

Rostovtseva T. S., Krasnoborov I. M., Krasnikov S. A. Chromosome numbers of some species of flora of Siberia / New data about phytogeography of Siberia [Novye dannye o fitogeographii Sibiri]. - Novosibirsk: Nauka, 1981. - P. 215-220 [In Russian]. (Ростовцева Т. С., Красноборов И. М., Красникова С. А. Числа хромосом некоторых видов флоры Сибири / Новые данные о фитогеографии Сибири. - Новосибирск: Наука, 1981. - С. 215-220).

Simonet M. Contribution à l'étude caryologique des Veronica // Compt. rend. Soc. biol., 1934. - Vol. 117(36). $1153 \mathrm{p}$.

Smirnov S., German D., Kosachev P., Dyachenko S. Addition to the flora of Mongolia // Turczaninowia, 2003. Vol. 6, iss. 4. - P. 11-21 [In Russian]. (Смирнов С. В., Герман Д. А., Косачев П. А., Дьяченко С. А. Дополнения к флоре Монголии // Turczaninowia, 2003. - Т. 6, вып. 4. - С. 11-21).

Stepanov N.V. Veronica taigischensis (Scrophulariaceae), a new species from the West Sayan (In Russian) // Bot. Journ. [Bot. Zhurn.], 1997. - Vol. 82, No. 9. - P. 92-95 [In Russian]. (Cmenaнов H. B. Veronica taigischensis (Scrophulariaceae) - новый вид из Западного Саяна // Бот. журн., 1997. - Т. 82, № 9. - С. 92-95).

Trávnícek B. Notes on the taxonomy of Pseudolysimachion sect. Pseudolysimachion (Scrophulariaceae) in Europe. I. P. incanum and P. spicatum // Preslia, 1998. - Vol. 70. - P. 193-223.

Trávnícek B. Taxa of the genus Pseudolysimachion (Scrophulariaceae) in the Czech Republic. I. Identification key to the species. Geographical distribution of species of sect. Longifolia // Preslia, 2000. - Vol. 72. - P. 411-439. 